\title{
Non-anonymous ballot aggregation: an axiomatic generalization of Approval Voting
}

\author{
Jorge Alcalde-Unzu* and Marc Vorsatz ${ }^{\dagger}$
}

October 8, 2013

\begin{abstract}
We study axiomatically situations in which the society agrees to treat voters with different characteristics distinctly. In this setting, we propose a set of intuitive axioms and show that they jointly characterize a new class of voting procedures, called Type-weighted Approval Voting. According to this family, each voter has a strictly positive and finite weight (the weight is necessarily the same for all voters with the same characteristics) and the alternative with the highest number of weighted votes is elected. The implemented voting procedure reduces to Approval Voting in case all voters are identical or the procedure assigns the same weight to all types. Using this idea, we also obtain a new characterization of Approval Voting.
\end{abstract}

Keywords: Approval Voting, Characterization, Anonymity.

\footnotetext{
${ }^{*}$ Corresponding author. Department of Economics, Public University of Navarre, Campus Arrosadia, 31006 Pamplona, Spain. Email: jorge.alcalde@unavarra.es. Financial support from the Spanish Ministry of Education and Science, through the project ECO2012-34202, and Fundación Ramón Areces is gratefully acknowledged.

†Departamento de Análisis Económico II, Universidad Nacional de Educación a Distancia (UNED), Paseo Senda del Rey 11, 28040 Madrid, Spain and Fundación de Estudios de Economía Aplicada (FEDEA), Calle Jorge Juan 46, 28001 Madrid, Spain. Email: mvorsatz@cee.uned.es. Financial support from the Spanish Ministry of Education and Science, through the project ECO2012-31985, and Fundación Ramón Areces is gratefully acknowledged.
} 
JEL-Number: D71. 


\section{Introduction}

Motivation There are many instances in which the members of a society or an institution vote in order to take a decision and each voter's impact on the outcome depends on her/his underlying characteristics. Examples include the EU Member Council or the IMF Board of Directors, where the weight of a country is determined by its population size or its stake, respectively (see, Tables 1 and 2 in the Appendix); management boards, where the vote of the CEO tends to count double in case of a tie; or hiring decisions in academic institutions, where the opinion of senior members is usually given more weight. From a theoretical point of view, this implies that voters are not treated equally and that existing axiomatic results on the question of which voting procedure to implement do not directly apply. It is consequently the aim of this study to complement the existing literature on axiomatic voting theory by suggesting a general class of voting procedures that is able to cover these kinds of situations.

The aggregation procedures discussed in the literature differ essentially in the type of information they take into account from the individual preferences. For example, Plurality Voting, the most widely used voting procedure, allows each individual to indicate only her most preferred alternative (and the alternative with most votes is elected). One common critique of Plurality Voting is that it may actually result in the election of the worst alternative for a majority of individuals even in single-winner elections. As a simple example, consider the case when there are three alternatives, two of which are very similar. Then, if the votes for the two similar alternatives are distributed equally, the third alternative may be elected even though a majority of the voters would prefer either of the other two alternatives.

Approval Voting, introduced by Brams and Fishburn (1978), has been ex- 
plicitly designed to overcome this drawback of Plurality Voting by allowing individuals to vote for (or approve of) as many alternatives as they wish to. As usual, the alternative with most votes wins the election. Recent evidence from field experiments by Laslier and van der Straten (2008) in France and Alós-Ferrer and Granić (2011) in Germany has shown that Approval Voting modifies the overall ranking of the alternatives and that it tends to elect the alternative that is most widely accepted in the population. This is the main reason why we deviate from using Plurality Voting as a benchmark and frame our analysis in the (more general and more complex) context when individuals can approve any number of alternatives.

Characterizations We are interested in general voting procedures that are operable in different voting environments in which the set of voters and the set of alternatives might vary. In particular, given a population of potential voters and a conceivable set of alternatives, a voting procedure should specify an outcome (a non-empty subset of the set of feasible alternatives) for every electorate (the individuals that indeed vote) and every set of feasible alternatives (the alternatives actually standing for election). We also assume that voters are partitioned into types according to some exogenous characteristics in such a way that individuals with equally relevant characteristics belong to the same type. In the examples of indirect democracy mentioned earlier, one can think of classifying voters into types in function of the number of people or the stake the voter represents. In problems of decision making in small groups, the voter's type could be associated with some of her personal characteristics such as seniority, age, etc.

In this setting, we consider a set of intuitive properties. First, we introduce two consistency properties that impose some structure on how the result of the voting procedure should be adapted when the set of alternatives 
or the set of individuals change: Consistency in alternatives, which is the analogue of Arrow's Choice Axiom, states that if the set of feasible alternatives is reduced yet some of the originally elected alternatives remain feasible, then exactly those alternatives have to be elected in the new situation; and Consistency in voters, which requires that if two disjoint electorates select a common set out of two feasible alternatives, then exactly this set has to be elected when the two electorates are assembled. Afterwards, we consider two symmetry properties: Symmetry across types, which means that voters of the same type have to be treated equally; and Symmetry across alternatives, which is the classical neutrality property. Finally, we add two well-known conditions: Faithfulness, which asks that if there is a single voter who approves $x$ but not $y$, then $x$ has to be elected whenever $x$ and $y$ are the only two feasible alternatives; and Continuity which, roughly speaking, states that no group of individuals should be able to always impose completely its opinion on the result of an election when joint with a sufficiently large electorate formed by many subgroups that agree among them on the set of alternatives that has to be selected.

Our first result, Theorem 1, shows that these properties fully characterize a general class of voting procedures that we will call Type-weighted Approval Voting. Each voting procedure of this family is associated with a vector of strictly positive and finite weights, one for each type of voter, and the winning alternative is the one with the highest number of weighted votes. If no discrimination across types have sense in a particular context, all weights should be equal and the voting procedure reduces to Approval Voting. Exploiting this fact, we show in our second result, Theorem 2, that if Symmetry across types is strengthened to the classical condition of Anonymity (Symmetry across voters), one essentially obtains a new characterization of Approval 
Voting in which Faithfulness and Continuity are eliminated as necessary requirements.

Related Literature Our work contributes to the existing literature on axiomatic voting theory. Roberts (1991) was the first to characterize Plurality Voting. Richelson (1978), Ching (1996), and Yeh (2008) also characterize the Plurality Rule, but as a social choice correspondence and not as a voting procedure; that is, in these studies, the domain is the Cartesian product of all linear orders on the set of alternatives. Fishburn (1978, 1979), Sertel (1988), Baigent and Xu (1991), Goodin and List (2006), Vorsatz (2007) and Sato (2013) provide different characterizations of Approval Voting. Alós-Ferrer (2006) shows that the properties in one of Fishburn's characterizations are not independent. Maniquet and Mongin (2013) study possible social welfare orderings corresponding to Approval Voting and characterize them by Arrow's conditions when preferences are dichotomous. Finally, Massó and Vorsatz (2008) and Alcalde-Unzu and Vorsatz (2009) introduce classes of voting procedures that generalize Approval Voting in natural ways. In Massó and Vorsatz (2008), the neutrality property is relaxed; in Alcalde-Unzu and Vorsatz (2009), the weight of a vote is a decreasing function in the number of approved alternatives.

One can think of Massó and Vorsatz (2008) and the characterization obtained in Theorem 1 as dual approaches that bear important similarities. Massó and Vorsatz (2008) relax neutrality and, as a result, characterize voting rules that assign different weights to alternatives. In this paper, we weaken the classical anonymity property and, as a consequence, weights are assigned to voters. However, there is still one important asymmetry that naturally occurs in the formal analysis. In Massó and Vorsatz (2008), the relative weight between two alternatives can be easily determined because it 
is known from the voting rule how many votes one alternative has to receive in order to compensate one vote to the other alternative. Yet, the construction of a weighted representation of a voting rule when anonymity is relaxed is more complicated. This is because adding one voter to an election has the effect that the particular weight of this voter has to be determined endogenously as well, and therefore, does not provide sufficient information of how to determine the relative weights of the other voters. Only the additional requirement that voters are divided into types and that there is an infinite population of potential voters of each type allows us to determine the relative weights.

Our second characterization, Theorem 2, also relates to the literature mentioned before. By working with a variable set of alternatives, contrary to the majority of studies found in the literature, we can naturally impose the property of Consistency in alternatives (which ultimately allows the decision maker to go forth and back between social choice and social welfare functions) in substitution of other properties. The only two other studies along the same line that characterize Approval Voting are Vorsatz (2007) and Sato (2013). The former characterizes Approval Voting in a dichotomous preference setting using strategy-proofness. The latter characterizes Approval Voting independently and simultaneously to this paper by using a very similar set of axioms to that imposed in Theorem 2 (see the detailed discussion in Section 3).

\section{Notation and Definitions}

We consider a setting with variable sets of voters and alternatives. Formally, let $X$ be a finite set of conceivable alternatives. Generic alternatives will be 
denoted by $x, y$, and $z$; subsets of $X$ by $S$ and $T$. The cardinality of $X$, $|X|$, is greater than or equal to $3 .^{1}$ The set of feasible alternatives $K$, the alternatives that are actually standing for an election, is a non-empty subset of $X$. Our analysis focuses on the idea that the individuals participating in the election may differ in their characteristics. To model this, we assume that there is a finite set of types $\Theta=\{1,2, \ldots, \theta\}$ and that for each type $t \in \Theta$, there is an infinite number of potential voters $I_{t}$. Hence, $I \equiv \bigcup_{t \in \Theta} I_{t}$ is the population of all potential voters. The individuals actually participating in an election, an electorate $N$, is a non-empty and finite subset of the population I. We will also make frequent use of the capital letters $A$ and $B$ to denote electorates.

Each individual $i \in I$ partitions the set of alternatives $X$ into two sets: $M_{i} \in 2^{X}$ and $\left(X \backslash M_{i}\right)$. The interpretation is that $M_{i}$ is the set of alternatives $i$ votes for (or approves of). Thus, we can describe the opinion of an individual $i$ by only referring to the set $M_{i}$. A profile $M=\left(M_{i}\right)_{i \in I} \in\left(2^{X}\right)^{I}$ is a list of all votes. Given a profile $M$ and an electorate $N$, a response profile $M_{N}=\left(M_{i}\right)_{i \in N} \in\left(2^{X}\right)^{N}$ is the $n$-tuple of votes coming from the electorate $N$ at profile $M$. Given the response profile $M_{N}$, the number of votes $x$ receives from the individuals of type $t$ who belong to the electorate $N$ is denoted by $G_{x}^{t}\left(M_{N}\right)$. Thus, $G_{x}\left(M_{N}\right)=\sum_{t \in \Theta} G_{x}^{t}\left(M_{N}\right)$ is the total number of votes $x$ gets at $M_{N}$.

Given a set of feasible alternatives $K$ and an electorate $N$, a voting rule $v^{K, N}:\left(2^{X}\right)^{I} \rightarrow\left(2^{K} \backslash \emptyset\right)$ selects for all profiles $M$ a non-empty set of feasible alternatives $v^{K, N}(M)$ with the property that for all $M, \bar{M} \in\left(2^{X}\right)^{I}$ such that

\footnotetext{
${ }^{1}$ If there are only two conceivable alternatives, all results of the paper hold true. The unique difference is that, when $|X|=2$, one of the axioms, Consistency in alternatives, is superfluous. This will become evident from the proofs.
} 
$M_{N}^{K}=\bar{M}_{N}^{K}, v^{K, N}(M)=v^{K, N}(\bar{M})$. We write $v^{K}\left(M_{N}\right)$ instead of $v^{K, N}(M)$. A voting procedure $\left\{v^{K, N}:\left(2^{X}\right)^{I} \rightarrow\left(2^{K} \backslash \emptyset\right)\right\}_{K \subseteq X, N \subset I}$ is a family of voting rules, one for every set of feasible alternatives $K$ and every electorate $N$. It is denoted by $v$. Given the voting procedure $v$ and a set of feasible alternatives $K$, the subfamily $\left\{v^{K, N}:\left(2^{X}\right)^{I} \rightarrow\left(2^{K} \backslash \emptyset\right)\right\}_{N \subset I}$ is denoted by $v^{K}$.

Next, we introduce some known voting procedures in this context.

Definition 1 The voting procedure $v$ is Approval Voting if for all sets of feasible alternatives $K \subseteq X$ and all electorates $N \subset I$, $x \in v^{K}\left(M_{N}\right)$ if and only if $G_{x}\left(M_{N}\right) \geq G_{y}\left(M_{N}\right)$ for all $y \in K$.

Definition 2 The voting procedure $v$ is Disapproval Voting if for all sets of feasible alternatives $K \subseteq X$ and all electorates $N \subset I$, $x \in v^{K}\left(M_{N}\right)$ if and only if $G_{x}\left(M_{N}\right) \leq G_{y}\left(M_{N}\right)$ for all $y \in K$.

As we have already outlined in the Introduction, the main objective of our study is to relax the anonymity assumption underlying Approval Voting. One natural way to achieve this goal is to treat individuals with the same type equally but to possibly discriminate between votes coming from individuals of distinct types. The family we introduce next conceptualizes this idea by assigning an exogenous (possibly different) weight to each type.

Definition 3 The voting procedure $v$ is a Type-weighted Approval Voting if there exists a vector of weights $\mathbf{p}=\left(p_{1}, p_{2}, \ldots, p_{\theta}\right) \in \mathbb{R}_{++}^{\theta}$ such that for all sets of feasible alternatives $K \subseteq X$ and all electorates $N \subset I$, $x \in v^{K}\left(M_{N}\right)$ if and only if $\sum_{t \in \Theta} p_{t} \cdot G_{x}^{t}\left(M_{N}\right) \geq \sum_{t \in \Theta} p_{t} \cdot G_{y}^{t}\left(M_{N}\right)$ for all $y \in K$. If $\mathbf{p}$ is such that $p_{s}=p_{t}$ for all $s, t \in \Theta$, then all voters are treated equally and the voting procedure coincides with Approval Voting. 


\section{Axioms and Characterizations}

In this section, we are going to present a characterization of all Typeweighted Approval Voting procedures and a new characterization of Approval Voting. We start by introducing several properties.

Since we allow in our analysis for variable sets of feasible alternatives and voters, we necessarily need two consistency conditions that establish how the selected set of alternatives adapts as either of these changes. The first property states that if the set of feasible alternatives is reduced and some of the alternatives that originally were selected remain feasible, then exactly those have to be selected in the new situation.

Consistency in Alternatives: The voting procedure $v$ is consistent in alternatives if for all feasible sets of alternatives $S \subset T \subseteq X$, all profiles $M \in\left(2^{X}\right)^{I}$, and all electorates $N \subset I$ such that $v^{T}\left(M_{N}\right) \cap S \neq \emptyset$,

$$
v^{S}\left(M_{N}\right)=v^{T}\left(M_{N}\right) \cap S
$$

The property of Consistency in alternatives is important because it allows us to reformulate the question of which alternatives to choose from each subset of alternatives to the question of how to order all alternatives of the universal set $X$. To say it differently, the problems of constructing a social choice function and a social welfare function become equivalent (see, Arrow 1959). This is the reason why we can restrict our attention in the remaining axioms to sets of feasible alternatives that only contain two alternatives.

The second consistency property, Consistency in voters, says that if two disjoint groups of voters elect some common alternatives from the set $\{x, y\}$, then exactly those alternatives have to be elected if the two groups are joined. 
This kind of property has been used in many characterizations of voting procedures and social choice functions; examples include, Smith (1973), Young (1974), Hansson and Sahlquist (1976), Fishburn (1978), Richelson (1978), Sertel (1988), Alós-Ferrer (2006), Massó and Vorsatz (2008), and AlcaldeUnzu and Vorsatz (2009).

CONSISTENCY IN vOTERS: The voting procedure $v$ is consistent in voters if for all alternatives $x, y \in X$, all profiles $M \in\left(2^{X}\right)^{I}$, and all disjoint electorates $A, B \subset I$ such that $v^{\{x, y\}}\left(M_{A}\right) \cap v^{\{x, y\}}\left(M_{B}\right) \neq \emptyset$,

$$
v^{\{x, y\}}\left(M_{A \cup B}\right)=v^{\{x, y\}}\left(M_{A}\right) \cap v^{\{x, y\}}\left(M_{B}\right) .
$$

To shorten our notation, we will say that a voting procedure $v$ is consistent if it is both consistent in alternatives and consistent in voters.

Next, we will introduce three symmetry conditions. The first one, Symmetry across alternatives, is the standard Neutrality condition. It states that if alternatives are permuted, then the set of elected alternatives has to be permuted accordingly. To introduce it formally, we need additional notation: given a permutation $\mu: X \rightarrow X$ and a pair of alternatives $\{x, y\}$, let $\mu\left(M_{N}\right)$ and $\mu\left(v^{\{x, y\}}\left(M_{N}\right)\right)$ be the response profile and the set of elected alternatives permuted according to $\mu$.

Symmetry ACROSS ALTERnAtives: The voting procedure $v$ is symmetric across alternatives (or neutral) if for all alternatives $x, y \in X$, all profiles $M \in\left(2^{X}\right)^{I}$, all electorates $N \subset I$, and all permutations $\mu: X \rightarrow X$,

$$
\mu\left(v^{\{x, y\}}\left(M_{N}\right)\right)=v^{\mu(\{x, y\})}\left(\mu\left(M_{N}\right)\right) .
$$

The second symmetry condition, Symmetry across types, relaxes the classical anonymity axiom according to which the result of the election should 
be invariant to permutations of voters. Here, we only require this symmetry condition to hold true if voters of the same type are permuted. To introduce it formally, we say that two response profiles $M_{A}$ and $M_{B}^{\prime}$ are isomorphic relative to $\{x, y\}$ if for all $t \in \Theta$, there is a permutation $\pi_{t}: I_{t} \cap A \rightarrow I_{t} \cap B$ such that $M_{\pi_{t}(i)}^{\prime} \cap\{x, y\}=M_{i} \cap\{x, y\}$.

SYMMETRY ACROSS TYPES: The voting procedure $v$ is symmetric across types (or type-wise anonymous) if for all alternatives $x, y \in X$ and all response profiles $M_{A}$ and $M_{B}^{\prime}$ that are isomorphic relative to $\{x, y\}$,

$$
v^{\{x, y\}}\left(M_{A}\right)=v^{\{x, y\}}\left(M_{B}^{\prime}\right)
$$

Finally, the last symmetry condition, Symmetry across voters, is the classical Anonymity axiom. To introduce it formally, we will say that two response profiles $M_{A}$ and $M_{B}^{\prime}$ are strongly isomorphic relative to $\{x, y\}$ if there is a permutation $\pi: A \rightarrow B$ such that $M_{\pi(i)}^{\prime} \cap\{x, y\}=M_{i} \cap\{x, y\}$.

Symmetry ACROSS voters: The voting procedure $v$ is symmetric across voters (or anonymous) if for all alternatives $x, y \in X$ and all response profiles $M_{A}$ and $M_{B}^{\prime}$ that are strongly isomorphic relative to $\{x, y\}$,

$$
v^{\{x, y\}}\left(M_{A}\right)=v^{\{x, y\}}\left(M_{B}^{\prime}\right)
$$

To shorten our notation, we will say that a voting procedure $v$ is weakly symmetric if it is both symmetric across alternatives and symmetric across types. We will say that it is symmetric if it is also symmetric across voters.

To introduce the next property, Continuity, consider an infinite number of disjoint electorates such that all of them only select the same alternative $x$ from the set $\{x, y\}$. Suppose also that there is another electorate $A$, disjoint from the other electorates, for which $y$ is the unique alternative elected 
from the set $\{x, y\}$. The idea of continuity is that if a sufficient number of electorates that elect $x$ are joined together with $A$, then alternative $x$ should be elected (but not necessarily excluding $y$ ). This condition eliminates, for example, dictatorship-like procedures that give an infinite weight to some type of voters or procedures that break ties in a lexicographic way.

Continuity: The voting procedure $v$ is continuous if for all alternatives $x, y \in X$, all profiles $M \in\left(2^{X}\right)^{I}$, all successions of disjoint electorates $\left\{N_{p}\right\}$ such that $v^{\{x, y\}}\left(N_{p}\right)=\{x\}$ for all $p \in \mathbb{N}$, and any other electorate $A$ for which $A \cap N_{p}=\emptyset$ for all $p \in \mathbb{N}$ and $v^{\{x, y\}}\left(M_{A}\right)=\{y\}$, there exists $k \in \mathbb{N}$ such that

$$
x \in v^{\{x, y\}}\left(M_{N_{1} \cup N_{2} \cup \ldots \cup N_{k} \cup A}\right) .
$$

In the literature, similar conditions to Continuity are found under the names of Archimedean Property or Overwhelming Majority; see, Smith (1973), Young (1975), Richelson (1978), Myerson (1996), or Alcalde-Unzu and Vorsatz (2009). ${ }^{2}$

Finally, we introduce a weak unanimity condition which establishes that getting more votes is desirable. The following property is a weak condition inspired by Fishburn (1978).

FAithfulness: The voting procedure $v$ is faithful if for all individuals $i \in I$ and all alternatives $x, y \in X$,

$$
M_{i}=\{x\} \Rightarrow v^{\{x, y\}}\left(M_{i}\right)=\{x\} .
$$

\footnotetext{
${ }^{2}$ The main differences between this version and others are: $(i)$ In other stronger versions, it is imposed that $v^{\{x, y\}}\left(M_{N_{1} \cup N_{2} \cup \ldots \cup N_{k} \cup A}\right)=\{x\}$ and/or that the requirement has to be satisfied also for any $k^{\prime}>k$; and $(i i)$ other versions require that the electorates of the sequence have to be isomorphic instead of requiring that they select the same set of alternatives.
} 
Our first and main result is a characterization of all Type-weighted Approval Voting procedures.

Theorem 1 The voting procedure $v$ is consistent, weakly symmetric, continuous, and faithful if and only if it is a Type-weighted Approval Voting.

The proof that the mentioned properties imply $v$ to be a Type-weighted Approval Voting is constructive and divided into several steps (see the Appendix). We now shortly explain the structure of the proof in order to facilitate its reading.

1. It is shown in Lemma 1 that consistency in voters and neutrality implies that if one individual either approves both $x$ and $y$ or neither of the two alternatives, then eliminating this individual from the electorate does not affect the result of the election in case $x$ and $y$ are the only two feasible alternatives.

2. Lemma 2 shows that consistency in voters and weak symmetry implies that if $x$ and $y$ receive the same number of votes from each type $t \in \Theta$, then both alternatives have to be elected if they are the only two feasible alternatives.

3. Lemma 3 establishes that consistency in voters, weak symmetry and previous lemmas implies that if alternatives $z$ and $w$ receive the same number of votes from each type $t \in \Theta$ under the response profile $M_{B}^{\prime}$ as alternatives $x$ and $y$, respectively, under the response profile $M_{A}$, then $z$ (respectively, $w$ ) is elected from the set $\{z, w\}$ at $M_{B}^{\prime}$ if and only if $x$ (respectively, $y$ ) is elected from the set $\{x, y\}$ at $M_{A}$.

4. We construct a binary relation $\succsim$ over vectors $\left(x_{1}, x_{2}, \ldots, x_{\theta}\right) \in \mathbb{N}^{\theta}$, interpreting each of the vectors as a possible combination of numbers 
of votes from each type. The binary relation represents which combinations of votes is better than another for an alternative to be selected by the voting procedure when there are only two alternatives standing for election.

5. Given consistency in voters, weak symmetry, continuity and Lemma 3, Lemma 4 proves that the triple $\left(\mathbb{N}^{\theta}, \succsim,+\right)$ is a closed extensive structure.

6. Using a result in Krantz et al. (1971) about closed extensive structures, we can deduce that the binary relation $\succsim$ can be computed by calculating the number of weighted votes of each alternative, where the weight of a vote depends on the type of the individual casting this vote. By faithfulness, all these weights are strictly positive.

7. Then, combining steps 4 to 6 , we deduce that the subfamily $\left\{v^{K}\right\}_{|K|=2}$ behaves as a Type-weighted Approval Voting rule. The exact value of the weights of each particular rule are obtained by observing in the results of the rule how many votes one alternative has to receive to compensate one vote for the other alternative.

8. Finally, by applying consistency in alternatives, we extend this argument to sets of alternatives of any size to show that $v$ is a Typeweighted Approval Voting procedure.

We also show in the Appendix that the properties used in Theorem 1 are independent, separating both consistency and weak symmetry in their respective parts.

Proposition 1 The properties in Theorem 1 are independent. 
At this point it is important to study the consequences of strengthening Symmetry across types to the classical Anonymity property (that is, to strengthen weak symmetry to symmetry). ${ }^{3}$ Since Approval Voting is the unique Type-weighted Approval Voting procedure that is also symmetric, we obviously obtain a characterization of Approval Voting. However, one important insight is that one can then, at the same time, eliminate the continuity axiom, a fact that has been shown independently and simultaneously to this paper by Sato (2013) using a different proof technique. ${ }^{4}$ But one can even go one step further as it is shown in our second theorem: if one strengthens weak symmetry to symmetry and eliminates faithfulness together with continuity, one still obtains essentially a characterization of Approval Voting. ${ }^{5}$ Before introducing the result, we need to define a non-degenerate voting procedure as a voting procedure that does not always select all available alternatives. ${ }^{6}$

Theorem 2 A non-degenerate voting procedure $v$ is consistent and symmetric if and only if it is Approval Voting or Disapproval Voting.

We say that Theorem 2 is essentially a characterization of Approval Voting because of the following reasoning: if individuals are rational, each voter $i \in I$

\footnotetext{
${ }^{3}$ We are very grateful to an anonymous referee for indicating this question to us.

${ }^{4}$ There are slight but non-essential differences across the two settings: (i) Sato (2013) defines all axioms for any possible size of the set of feasible alternatives, whereas we define the properties for sets with only two feasible alternatives, except Consistency in alternatives; (ii) Sato (2013) considers an independent property called Consistency in ballot response profiles, an assumption that we included in the definition of the voting procedure; and (iii) We impose a condition of non-degeneration in our next result that it is not needed when faithfulness is added as a necessary axiom.

${ }^{5}$ We omit the independence of the axioms given that it is very similar to the one of Theorem 1 .

${ }^{6}$ Formally, $v$ is a non-degenerate voting procedure if there exists a set of alternatives $K \subseteq X$, a set of voters $N \subset I$ and a profile $M \in\left(2^{X}\right)^{I}$ such that $v^{K}\left(M_{N}\right) \neq K$.
} 
will permute the sets $M_{i}$ and $\left(X \backslash M_{i}\right)$ if Disapproval Voting is implemented instead of Approval Voting. Thus, the results of the two voting procedures are always the same for rational voters. Yet, if one wants to isolate Approval Voting in its classical definition, an additional requirement satisfied by Approval but not by Disapproval Voting becomes necessary. Some possibilities are Faithfulness (as shown by Sato, 2013), any other unanimity property, any monotonicity condition, or even a No-Disapproval Voting axiom: a minimal property that only eliminates Disapproval Voting from the set of possible voting procedures. ${ }^{7}$

The proof that the mentioned properties imply $v$ to be Approval Voting or Disapproval Voting is constructive and similar to that of Theorem 1 (see the Appendix). In particular, step 1 is the same in both proofs, step 2 is not necessary any further, and the remaining steps follow a similar path, but with strong results in this case given that the set of properties is stronger. We now shortly explain this final part in order to facilitate the reading of the proof.

3. Lemma 5 shows, applying Lemma 1, that under consistency and symmetry if alternatives $z$ and $w$ receive the same number of total votes under the response profile $M_{B}^{\prime}$ as alternatives $x$ and $y$, respectively, under the response profile $M_{A}$, then $z$ (respectively, $w$ ) is elected from the set $\{z, w\}$ at $M_{A}$ if and only if $x$ (respectively, $y$ ) is elected from the set $\{x, y\}$ at $M_{B}^{\prime}$.

4. We construct a binary relation $\succsim$ over $\mathbb{N}$. The binary relation represents which number of votes is better than another for an alternative to

\footnotetext{
${ }^{7}$ This is the weakest possible axiom because it only eliminates one voting procedure from the analysis. A similar axiom, but in a totally different context, was proposed by D'Aspremont and Gevers (1977) to isolate the leximin principle from the leximax one.
} 
be selected by the voting procedure when there are only two feasible alternatives.

5. Lemma 6 shows, using Consistency in voters, symmetry and Lemma 5, that $\succsim$ corresponds with $\geq, \leq$ or $=$. Hence, the subfamily $\left\{v^{K}\right\}_{|K|=2}$ behaves as Approval Voting $(\geq)$, as Disapproval Voting $(\leq)$ or as a degenerate voting procedure $(=)$.

6. Using Consistency in alternatives, we extend this argument to sets of alternatives of any size. Thus, if $v$ is non-degenerate, it is Approval or Disapproval Voting.

The main difference between this characterization of Approval Voting (and the one by Sato, 2013) with respect to other known characterizations is that we work with a variable set of alternatives instead of a fixed one, as other papers do. This fact allows, apart of the possibility of not including a monotonicity property, to include Consistency in alternatives replacing other axioms that are perhaps hinting too direct to the resulting voting procedure. For example, the property of Cancellation — which requires that all alternatives are elected whenever all alternatives receive the same number of votes —, used in Fishburn (1978) and in Alós-Ferrer (2006), practically imposes that only the total number of votes matter. Similarly, Baigent and Xu (1991) and Goodin and List (2006) suggest a property that ensures that the result of the voting procedure does not change if we permute the individual who cast a vote for a given alternative. A final example along this line is the property of Disjoint Equality, considered by Fishburn (1978) and Sertel (1988), which states that if two voters approve disjoint sets of alternatives, then the electorate composed by the two voters selects the union of these two approved sets. These axioms are well-founded, yet Consistency in alternatives - Ar- 
row's axiom of choice, which is a necessary and sufficient condition so that no information is distorted if a transitive and complete binary relation is derived from the pairwise choices of a given social choice function and, afterwards, the social choice function is re-derived as the top indifference class of the derived social welfare function restricted to the set of available alternatives - seems to us a far more basic property.

The imposition of Consistency in alternatives also allows us to directly relate our characterization to existing results on social welfare functions. ${ }^{8}$ For example, Ju (2011) shows that if preferences are dichotomous, then all monotonic, neutral, and anonymous social welfare orderings are acyclic and the majority rule is the unique non-degenerate one that is transitive. Since one can reinterpret the alternatives $M_{i}$ an individual $i$ votes for as the set of good alternatives and the alternatives an individual does not vote for as the set of bad alternatives of a dichotomous preference relation, there is an obvious equivalence between our framework and the domain restriction Ju (2011) imposes and, therefore, between Approval Voting and the majority rule. Using this interpretation, since faithfulness and consistency in voters imply the monotonicity condition of $\mathrm{Ju}$ (2011), Theorem 1 shows that there are additional transitive social welfare orderings once the anonymity assumption is relaxed. Similarly, Theorem 2 shows that the substitution of monotonicity by Consistency in voters also allows us to characterize essentially the majority rule under a dichotomous domain restriction.

\footnotetext{
${ }^{8}$ We thank an anonymous referee for drawing our attention to this discussion.
} 


\section{Conclusion}

We have characterized a family of voting procedures that generalizes Approval Voting for the cases when the society agrees not to treat all voters equally. In particular, we have shown that a voting procedure is consistent, weakly symmetric, continuous, and faithful if and only if there exists a strictly positive and finite weight for each type of voter and the alternatives with the maximal sum of weighted votes are selected.

Since we characterize a large class of voting rules and therefore allow a priori for a wide variety of discrimination between voters, there is the need to discuss how the weights are ultimately determined. In particular, one needs to identify first the relevant characteristics of the voters. Only afterwards one has to decide how to weigh different voters.

In some cases of indirect democracy in which each voter represents a set of citizens, the first step is not controversial: the classification should be done in function of the number of people each voter represents. However, there is a vast literature that discusses which weight each representative should have as a function of her type. At a first sight, one would think that the weights should be proportional to the number of people each voter represents. Yet, it has been shown that this is probably not the best voting rule. Barbera and Jackson (2006) characterize the efficient weights -the ones that maximize the total expected utility-, which turn out to be different from the proportional ones. Other authors have proposed also structures of weights in basis of other criteria such as the equality of the probability of each person to be pivotal in the election or the equality of the expected satisfaction of each person with the outcome. See, for example, Laruelle and Valenciano (2008) for this strand of the literature.

In many other cases the voters only represent themselves, yet it might 
still be desirable to implement a discriminative voting rule. This is the point where the discussion about which characteristics should determine the weight clearly emerges. Even though it is impossible to provide a definite guideline, one can look, for example, at elections in universities. In some of them, voters are classified depending on their type of affiliation (students, administrative staff, professors, etc.); in others, the classification is more detailed and also considers other aspects such as seniority.

All in all, and independently of the more or less difficulty to define the appropriate criteria to classify voters, our axiomatic study provides a theoretical background for the use of a Type--weighted Approval Voting procedure.

\section{References}

1. Alcalde-Unzu J and M Vorsatz (2009). Size Approval Voting. Journal of Economic Theory 119: 1187-1210.

2. Alós-Ferrer C (2006). A Simple Characterization of Approval Voting. Social Choice and Welfare 27: 621-625.

3. Alós-Ferrer C and D Granić (2011). Two Field Experiments of Approval Voting in Germany. Social Choice and Welfare, forthcoming.

4. Arrow K (1959). Rational choice functions and orderings. Economica 26: $121-127$.

5. Baigent N and Xu Y (1991). Independent Necessary and Sufficient Conditions for Approval Voting. Mathematical Social Sciences 21: 2129. 
6. Barberà S and M Jackson (2006). On the Weights of Nations: Assigning Voting Weights in a Heterogeneous Union. Journal of Political Economy 114: 317-339.

7. Brams S and P Fishburn (1978). Approval Voting. American Political Science Review 72: 831-847.

8. Ching S (1996). A Simple Characterization of Plurality Rule. Journal of Economic Theory 71: 298-302.

9. D'Aspremont C and L Gevers (1977) Equity and the Informational Basis of Collective Choice. Review of Economic Studies 44: 199-209.

10. Hansson B and H Sahlquist (1976). A Proof Technique for Social Choice with Variable Electorates. Journal of Economic Theory 13: 193-200.

11. Fishburn P (1978). Axioms for Approval Voting: Direct Proof. Journal of Economic Theory 19: 180-185.

12. Fishburn P (1979). Symmetric and Consistent Aggregation with Dichotomous Voting. In: Aggregation and Revelation of Preferences (ed. JJ Laffont). North-Holland, Amsterdam.

13. Goodin R and C List (2006). A Conditional Defense of Plurality Rule: Generalizing May's Theorem in a Restricted Informational Environment. American Journal of Political Science 50: 940-949.

14. Ju B (2011). Collectively rational voting rules for simple preferences. Journal of Mathematical Economics 47: 143-149. 
15. Krantz D, R Luce, P Suppes, and A Tversky (1971). Foundations of Measurement I: Additive and Polynomial Representations. Academic Press, New York.

16. Laslier JF and K van der Straeten (2008). A Live Experiment on Approval Voting. Experimental Economics 11: 97-105.

17. Laruelle A and F Valenciano (2008). Voting and Collective DecisionMaking: Bargaining and Power. Cambridge University Press, Cambridge, New York.

18. Maniquet F and P Mongin (2013). Approval Voting and Arrow's Impossibility Theorem. Working Paper. Available at SSRN:

http://ssrn.com/abstract $=2239118$

19. Massó J and M Vorsatz (2008). Weighted Approval Voting. Economic Theory 36: 129-146.

20. Myerson R (1996). Axiomatic Derivation of Scoring Rules without the Ordering Assumption. Social Choice and Welfare 12: 59-74.

21. Richelson J (1978). A Characterization Result for the Plurality Rule. Journal of Economic Theory 19: 548-550.

22. Roberts F (1991). Characterizations of the Plurality Function. Mathematical Social Sciences 21: 101-127.

23. Sato N (2013) A characterization result for approval voting with a variable set of alternatives. Working paper. Available at SSRN: http://ssrn.com/abstract $=2232922$.

24. Sertel M (1988). Characterizing Approval Voting. Journal of Economic Theory 45: 207-211. 
25. Smith H (1973). Aggregation of Preferences with Variable Electorates. Econometrica 41: 1027-1041.

26. Vorsatz M (2007). Approval Voting on Dichotomous Preferences. Social Choice and Welfare 28: 127-141.

27. Yeh C (2008). An Efficiency Characterization of Plurality Rule in Collective Choice Problems. Economic Theory 34: 575-583.

28. Young P (1974). An Axiomatization of Borda's Rule. Journal of Economic Theory 9: 43-52.

29. Young P (1975). Social Choice Scoring Functions. SIAM Journal of Applied Mathematics 28: 824-838. 


\section{Proofs}

The proofs of Theorems 1 and 2 have the following lemma in common.

Lemma 1 If the voting procedure $v$ is consistent in voters and neutral, then for all alternatives $x, y \in X$, all profiles $M \in\left(2^{X}\right)^{I}$, all electorates $N \subset I$ and all voters $i \in N$ such that $M_{i} \cap\{x, y\} \in\{\emptyset,\{x, y\}\}$,

$$
v^{\{x, y\}}\left(M_{N}\right)=v^{\{x, y\}}\left(M_{N \backslash\{i\}}\right) .
$$

Proof: Take any two alternatives $x, y \in X$, any profile $M \in\left(2^{X}\right)^{I}$, any electorate $N \subset I$ and any voter $i \in N$ such that $M_{i} \cap\{x, y\} \in\{\emptyset,\{x, y\}\}$. We are going to show first by contradiction that $v^{\{x, y\}}\left(M_{i}\right)=\{x, y\}$.

Suppose that $v^{\{x, y\}}\left(M_{i}\right)=\{x\}$. Consider the permutation $\mu: X \rightarrow X$ such that $\mu(x)=y, \mu(y)=x$ and $\mu(z)=z$ for all $z \in X \backslash\{x, y\}$. Then, by neutrality, $\mu\left(v^{\{x, y\}}\left(M_{i}\right)\right)=v^{\mu(\{x, y\})}\left(\mu\left(M_{i}\right)\right)$. Given that $\mu\left(v^{\{x, y\}}\left(M_{i}\right)\right)=\{y\}$, that $\mu(\{x, y\})=\{x, y\}$ by definition of $\mu$ and that $\mu\left(M_{i}\right)=M_{i}$, we have that $v^{\{x, y\}}\left(M_{i}\right)=\{y\}$. This is a contradiction. Since $v^{\{x, y\}}\left(M_{i}\right)=\{y\}$ can be excluded using a similar argument and since $v^{\{x, y\}}\left(M_{i}\right) \neq \emptyset$ by definition, we can conclude that $v^{\{x, y\}}\left(M_{i}\right)=\{x, y\}$. Finally, $v^{\{x, y\}}\left(M_{N \backslash\{i\}}\right) \cap\{x, y\} \neq \emptyset$ implies that we can apply consistency in voters to obtain that $v^{\{x, y\}}\left(M_{N}\right)=$ $v^{\{x, y\}}\left(M_{N \backslash\{i\}}\right)$. This concludes the proof of the lemma.

The successive applications of Lemma 1 implies that given an electorate $N$ and any two alternatives $x$ and $y$ standing for election, it can be assumed that all individuals belonging to $N$ vote for one and only one of these two alternatives (voters who do not declare a strict preference between $x$ and $y$ can simply be discarded). Also note that if $M_{i} \in\{\emptyset,\{x, y\}\}$ for all $i \in N$, then both alternatives have to be elected by neutrality. 


\section{Proof of Theorem 1}

It is easy to check that all Type-weighted Approval Voting procedures satisfy consistency, weak symmetry, continuity and faithfulness. The proof that these properties imply $v$ to be a Type-weighted Approval Voting follows the steps mentioned in the main text.

We first have that Lemma 1 can be applied. Now, we establish other lemmas.

Lemma 2 If the voting procedure $v$ is consistent in voters and weakly symmetric, then for all alternatives $x, y \in X$, all profiles $M \in\left(2^{X}\right)^{I}$, and all electorates $N \subset I$ such that $G_{x}^{t}\left(M_{N}\right)=G_{y}^{t}\left(M_{N}\right)$ for all $t \in \Theta$,

$$
v^{\{x, y\}}\left(M_{N}\right)=\{x, y\} .
$$

Proof: Take any two alternatives $x, y \in X$, any profile $M \in\left(2^{X}\right)^{I}$, and any electorate $N \subset I$ such that $G_{x}^{t}\left(M_{N}\right)=G_{y}^{t}\left(M_{N}\right)$ for all $t \in \Theta$. By Lemma 1 , we can assume that $M_{i} \cap\{x, y\} \in\{\{x\},\{y\}\}$ for all $i \in N$. Partition the electorate $N$ into $\theta$ sub-electorates $N_{1}, \ldots, N_{\theta}$ in such a way that $i \in N_{t}$ if and only if $i \in N \cap I_{t}$.

Consider any type $t \in \Theta$ for which $\left|N_{t}\right|>0$. We are going to show by contradiction that $v^{\{x, y\}}\left(M_{N_{t}}\right)=\{x, y\}$. Suppose that $v^{\{x, y\}}\left(M_{N_{t}}\right)=\{x\}$. Take the permutation $\mu: X \rightarrow X$ such that $\mu(x)=y, \mu(y)=x$, and $\mu(z)=z$ for all $z \in X \backslash\{x, y\}$. Then, by neutrality, $v^{\mu(\{x, y\})}\left(\mu\left(M_{N_{t}}\right)\right)=\mu\left(v^{\{x, y\}}\left(M_{N_{t}}\right)\right)=$ $\{y\}$. Since $\mu(\{x, y\})=\{x, y\}$ by the definition of the permutation, the former equation can be rewritten as $v^{\{x, y\}}\left(\mu\left(M_{N_{t}}\right)\right)=\{y\}$. Now observe that $\mu\left(M_{N_{t}}\right)$ is an isomorphic copy of $M_{N_{t}}$ relative to $\{x, y\}$ because $G_{x}^{t}\left(M_{N}\right)=G_{y}^{t}\left(M_{N}\right)$ by assumption. By type-wise anonymity, $v^{\{x, y\}}\left(M_{N_{t}}\right)=v^{\{x, y\}}\left(\mu\left(M_{N_{t}}\right)\right)=$ $\{y\}$, which contradicts our initial assumption that $v^{\{x, y\}}\left(M_{N_{t}}\right)=\{x\}$. A symmetric argument proves that $v^{\{x, y\}}\left(M_{N_{t}}\right) \neq\{y\}$ and, therefore, we are able to conclude that $v^{\{x, y\}}\left(M_{N_{t}}\right)=\{x, y\}$. 
Finally, using that $\bigcap_{t \in \Theta:\left|N_{t}\right|>0} v^{\{x, y\}}\left(M_{N_{t}}\right) \neq \emptyset$, the iterative application of consistency in voters implies that

$$
v^{\{x, y\}}\left(M_{N}\right)=v^{\{x, y\}}\left(\sum_{t \in \Theta:\left|N_{t}\right|>0} M_{N_{t}}\right)=\bigcap_{t \in \Theta:\left|N_{t}\right|>0} v^{\{x, y\}}\left(M_{N_{t}}\right)=\{x, y\} .
$$

This concludes the proof of the lemma.

Before introducing the next lemma, let $d_{x, y}^{t}\left(M_{N}\right)=G_{x}^{t}\left(M_{N}\right)-G_{y}^{t}\left(M_{N}\right)$ be the difference of the number of votes of type $t$ between alternatives $x$ and $y$ at $M_{N}$.

Lemma 3 If the voting procedure $v$ is consistent in voters and weakly symmetric, then for all alternatives $x, y, z, w \in X$, all profiles $M, M^{\prime} \in\left(2^{X}\right)^{I}$, and all electorates $A, B \subset I$ such that $G_{x}^{t}\left(M_{A}\right)=G_{z}^{t}\left(M_{B}^{\prime}\right)$ and $G_{y}^{t}\left(M_{A}\right)=$ $G_{w}^{t}\left(M_{B}^{\prime}\right)$ for all $t \in \Theta$,

$$
x \in v^{\{x, y\}}\left(M_{A}\right) \Leftrightarrow z \in v^{\{z, w\}}\left(M_{B}^{\prime}\right) \text { and } y \in v^{\{x, y\}}\left(M_{A}\right) \Leftrightarrow w \in v^{\{z, w\}}\left(M_{B}^{\prime}\right)
$$

Proof: Take any four alternatives $x, y, z, w \in X$, any two profiles $M, M^{\prime} \in$ $\left(2^{X}\right)^{I}$, and any two electorates $A, B \subset I$ such that $G_{x}^{t}\left(M_{A}\right)=G_{z}^{t}\left(M_{B}^{\prime}\right)$ and $G_{y}^{t}\left(M_{A}\right)=G_{w}^{t}\left(M_{B}^{\prime}\right)$ for all $t \in \Theta$. By Lemma 1, we can assume that $M_{i} \cap\{x, y\} \in\{\{x\},\{y\}\}$ for all $i \in A$ and $M_{j}^{\prime} \cap\{x, y\} \in\{\{x\},\{y\}\}$ for all $j \in B$. Partition the electorate $A$ into $\theta$ sub-electorates $A_{1}, \ldots, A_{\theta}$ in such a way that $i \in A_{t}$ if and only if $i \in A \cap I_{t}$. Construct the electorates $B_{1}, \ldots, B_{\theta}$ in an identical manner.

For each type $t \in \Theta$, partition the electorate $A_{t}$ into two sub-electorates, $A_{t_{1}}$ and $A_{t_{2}}$, in such a way that exactly $\left|d_{x, y}^{t}\left(M_{A}\right)\right|$ individuals belong to $A_{t_{1}}$ and all these individuals only vote for the alternative that receives more votes at $M_{A}$; that is, for all $i \in A_{t_{1}}, M_{i} \cap\{x, y\}=\{x\}$ whenever $G_{x}^{t}\left(M_{A}\right)>G_{y}^{t}\left(M_{A}\right)$ and $M_{i} \cap\{x, y\}=\{y\}$ whenever $G_{x}^{t}\left(M_{A}\right)<G_{y}^{t}\left(M_{A}\right)$ and, obviously, $A_{t_{1}}=\emptyset$ 
in case $G_{x}^{t}\left(M_{A}\right)=G_{y}^{t}\left(M_{A}\right)$. Then, $A_{t_{2}}=A_{t} \backslash A_{t_{1}}$. The electorates $B_{t_{1}}$ and $B_{t_{2}}$ are derived from $B_{t}$ in a similar fashion.

Consider the permutation $\mu: X \rightarrow X$ such that $\mu(z)=x$ and $\mu(w)=y$. Then, $\mu\left(M_{B_{t_{j}}}^{\prime}\right)$ and $M_{A_{t_{j}}}$ are isomorphic relative to $\{x, y\}$ for all $t \in \Theta$ and all $j \in\{1,2\}$. Summing up over all types we can see that the response profiles $M_{A_{1}}=\sum_{t \in \Theta:\left|A_{t_{1}}\right|>0} M_{A_{t_{1}}}$ and $\mu\left(M_{B_{1}}^{\prime}\right)=\sum_{t \in \Theta:\left|B_{t_{1}}\right|>0} \mu\left(M_{B_{t_{1}}}^{\prime}\right)$ are isomorphic relative to $\{x, y\}$. By type-wise anonymity,

$$
v^{\{x, y\}}\left(M_{A_{1}}\right)=v^{\{x, y\}}\left(\mu\left(M_{B_{1}}^{\prime}\right)\right)
$$

Also, we have that $d_{x, y}^{t}\left(M_{A_{t_{2}}}\right)=d_{z, w}^{t}\left(M_{B_{t_{2}}}^{\prime}\right)=0$ for all $t \in \Theta$ by the way we partitioned the electorates. So, define $M_{A_{2}}=\sum_{t \in \Theta:\left|A_{t_{2}}\right|>0} M_{A_{t_{2}}}$ and $\mu\left(M_{B_{2}}^{\prime}\right)=\sum_{t \in \Theta:\left|B_{t_{2}}\right|>0} \mu\left(M_{B_{t_{2}}}^{\prime}\right)$ and apply Lemma 2 to see that

$$
v^{\{x, y\}}\left(M_{A_{2}}\right)=v^{\{x, y\}}\left(\mu\left(M_{B_{2}}^{\prime}\right)\right)=\{x, y\} .
$$

Now, apply consistency in voters together with Equations (1) and (2) to see that $v^{\{x, y\}}\left(M_{A}\right)=v^{\{x, y\}}\left(M_{A_{1} \cup A_{2}}\right)=v^{\{x, y\}}\left(M_{A_{1}}\right)$ and that $v^{\{x, y\}}\left(\mu\left(M_{B}^{\prime}\right)\right)=$ $v^{\{x, y\}}\left(\mu\left(M_{B_{1} \cup B_{2}}^{\prime}\right)\right)=v^{\{x, y\}}\left(\mu\left(M_{B_{1}}^{\prime}\right)\right)$. This, together with Equation (1), implies that

$$
v^{\{x, y\}}\left(M_{A}\right)=v^{\{x, y\}}\left(\mu\left(M_{B}^{\prime}\right)\right) .
$$

Finally, consider the permutation $\mu^{-1}$. By neutrality, $\mu^{-1}\left(v^{\{x, y\}}\left(\mu\left(M_{B}^{\prime}\right)\right)\right)=$ $v^{\mu^{-1}(\{x, y\})}\left(\mu^{-1}\left(\mu\left(M_{B}^{\prime}\right)\right)\right)=v^{\{z, w\}}\left(M_{B}^{\prime}\right)$. This, together with Equation (3), implies that $\mu^{-1}\left(v^{\{x, y\}}\left(M_{A}\right)\right)=v^{\{z, w\}}\left(M_{B}^{\prime}\right)$. Hence, $x \in v^{\{x, y\}}\left(M_{A}\right)$ if and only if $z \in v^{\{z, w\}}\left(M_{B}^{\prime}\right)$ and $y \in v^{\{x, y\}}\left(M_{A}\right)$ if and only if $w \in v^{\{z, w\}}\left(M_{B}^{\prime}\right)$.

Consider the following binary relation $\succsim$ defined over $\mathbb{N}^{\theta} \times \mathbb{N}^{\theta}$ : for all $\left(x_{1}, \ldots, x_{\theta}\right),\left(y_{1}, \ldots, y_{\theta}\right) \in \mathbb{N}^{\theta},\left(x_{1}, \ldots, x_{\theta}\right) \succsim\left(y_{1}, \ldots, y_{\theta}\right)$ if there exists a response profile $M_{N}$ and two alternatives $x, y \in X$ such that $x \in v^{\{x, y\}}\left(M_{N}\right)$ and for all $t \in \Theta, G_{x}^{t}\left(M_{N}\right)=x_{t}$ and $G_{y}^{t}\left(M_{N}\right)=y_{t}$. Our objective is to show 
that the triple $\left(\mathbb{N}^{\theta}, \succsim,+\right)$ is a closed extensive structure; that is, this triple satisfies the following properties (see Krantz et al. 1971):

1. Complete PREORDER: $\succsim$ is a complete preorder over $\mathbb{N}^{\theta} \times \mathbb{N}^{\theta}$.

2. Associativity: For all $\left(x_{1}, \ldots, x_{\theta}\right),\left(y_{1}, \ldots, y_{\theta}\right),\left(z_{1}, \ldots, z_{\theta}\right) \in \mathbb{N}^{\theta}$, we have that $\left(x_{1}, \ldots, x_{\theta}\right)+\left(\left(y_{1}, \ldots, y_{\theta}\right)+\left(z_{1}, \ldots, z_{\theta}\right)\right) \sim\left(\left(x_{1}, \ldots, x_{\theta}\right)+\right.$ $\left.\left(y_{1}, \ldots, y_{\theta}\right)\right)+\left(z_{1}, \ldots, z_{\theta}\right)$.

3. IndePendence: For all $\left(x_{1}, \ldots, x_{\theta}\right),\left(y_{1}, \ldots, y_{\theta}\right),\left(z_{1}, \ldots, z_{\theta}\right) \in \mathbb{N}^{\theta}$, we have that $\left(x_{1}, \ldots, x_{\theta}\right) \succsim\left(y_{1}, \ldots, y_{\theta}\right) \Leftrightarrow\left(\left(x_{1}, \ldots, x_{\theta}\right)+\left(z_{1}, \ldots, z_{\theta}\right)\right) \succsim$ $\left(\left(y_{1}, \ldots, y_{\theta}\right)+\left(z_{1}, \ldots, z_{\theta}\right)\right) \Leftrightarrow\left(\left(z_{1}, \ldots, z_{\theta}\right)+\left(x_{1}, \ldots, x_{\theta}\right)\right) \succsim\left(\left(z_{1}, \ldots, z_{\theta}\right)+\right.$ $\left.\left(y_{1}, \ldots, y_{\theta}\right)\right)$.

4. Archimedean: For all four $\left(x_{1}, \ldots, x_{\theta}\right),\left(y_{1}, \ldots, y_{\theta}\right),\left(z_{1}, \ldots, z_{\theta}\right)$ and $\left(w_{1}, \ldots, w_{\theta}\right) \in \mathbb{N}^{\theta}$, if $\left(x_{1}, \ldots, x_{\theta}\right) \succ\left(y_{1}, \ldots, y_{\theta}\right)$, then there exists a positive integer $t$ such that $\left(k \cdot\left(x_{1}, \ldots, x_{\theta}\right)+\left(z_{1}, \ldots, z_{\theta}\right)\right) \succsim\left(k \cdot\left(y_{1}, \ldots, y_{\theta}\right)+\right.$ $\left.\left(w_{1}, \ldots, w_{\theta}\right)\right)$.

Lemma 4 If the voting procedure $v$ is consistent in voters, weakly symmetric and continuous, the triple $\left(\mathbb{N}^{\theta}, \succsim,+\right)$ is a closed extensive structure.

Proof: We show that the triple $\left(\mathbb{N}^{\theta}, \succsim,+\right)$ satisfies the conditions of Complete Preorder, Associativity, Independence, and Archimedean.

Complete Preorder: To see that the binary relation $\succsim$ is well-defined, take any $\left(x_{1}, \ldots, x_{\theta}\right),\left(y_{1}, \ldots, y_{\theta}\right) \in \mathbb{N}^{\theta}$ and consider the response profiles $M_{A}, M_{B}^{\prime}$ together with the alternatives $x, y, z, w \in X$ such that $G_{x}^{t}\left(M_{A}\right)=$ $G_{z}^{t}\left(M_{B}^{\prime}\right)=x_{t}$ and $G_{y}^{t}\left(M_{A}\right)=G_{w}^{t}\left(M_{B}^{\prime}\right)=y_{t}$ for all $t \in \Theta$. We have to establish that $x \in v^{\{x, y\}}\left(M_{A}\right) \Leftrightarrow z \in v^{\{z, w\}}\left(M_{B}^{\prime}\right)$ and that $y \in v^{\{x, y\}}\left(M_{A}\right) \Leftrightarrow$ 
$w \in v^{\{z, w\}}\left(M_{B}^{\prime}\right)$. But this is exactly what we have shown in Lemma 3. Hence, $\succsim$ is well-defined.

To show that the binary relation $\succsim$ is complete, note first that for any $\left(x_{1}, \ldots, x_{\theta}\right),\left(y_{1}, \ldots, y_{\theta}\right) \in \mathbb{N}^{\theta}$, we can consider a response profile $M_{N}$ and two alternatives $x, y \in X$ such that for all $t \in \Theta, N \cap I_{t}$ consists of $x_{t}$ individuals voting only for alternative $x$ and $y_{t}$ individuals voting only for alternative $y$. This is always possible because $I_{t}$ is an infinite set for all $t \in \Theta$. By definition of $v$, we have that $v^{\{x, y\}}\left(M_{N}\right) \in\{\{x\},\{y\},\{x, y\}\}$. Then, it follows from the definition of $\succsim$ that $\left(x_{1}, \ldots, x_{\theta}\right) \succsim\left(y_{1}, \ldots, y_{\theta}\right)$ and/or $\left(y_{1}, \ldots, y_{\theta}\right) \succsim\left(x_{1}, \ldots, x_{\theta}\right)$. Hence, $\succsim$ is complete.

To see that $\succsim$ is transitive, take any $\left(x_{1}, \ldots, x_{\theta}\right),\left(y_{1}, \ldots, y_{\theta}\right),\left(z_{1}, \ldots, z_{\theta}\right) \in$ $\mathbb{N}^{\theta}$ such that $\left(x_{1}, \ldots, x_{\theta}\right) \succsim\left(y_{1}, \ldots, y_{\theta}\right)$ and $\left(y_{1}, \ldots, y_{\theta}\right) \succsim\left(z_{1}, \ldots, z_{\theta}\right)$. Consider any response profile $M_{N}$ and any three alternatives $x, y, z \in X$ such that for all $t \in \Theta, N \cap I_{t}$ consists of $x_{t}$ individuals voting only for alternative $x, y_{t}$ individuals voting only for alternative $y$, and $z_{t}$ individuals voting only for alternative $z$. Since $\left(x_{1}, \ldots, x_{\theta}\right) \succsim\left(y_{1}, \ldots, y_{\theta}\right)$ and $\left(y_{1}, \ldots, y_{\theta}\right) \succsim\left(z_{1}, \ldots, z_{\theta}\right)$ by assumption, the definition of $\succsim$ implies that $x \in v^{\{x, y\}}\left(M_{N}\right)$ and $y \in v^{\{y, z\}}\left(M_{N}\right)$. Suppose that transitivity is violated; that is, $v^{\{x, z\}}\left(M_{N}\right)=\{z\}$. Then,

(a) $x \notin v^{\{x, y, z\}}\left(M_{N}\right)$. If it was the case that $x \in v^{\{x, y, z\}}\left(M_{N}\right)$, then, by consistency in alternatives, we would have $x \in v^{\{x, z\}}\left(M_{N}\right)$. This contradicts $v^{\{x, z\}}\left(M_{N}\right)=\{z\}$.

(b) $y \notin v^{\{x, y, z\}}\left(M_{N}\right)$. If it was the case that $y \in v^{\{x, y, z\}}\left(M_{N}\right)$, then, by consistency in alternatives, we would have $y \in v^{\{x, y\}}\left(M_{N}\right)$. This, together with the assumption $x \in v^{\{x, y\}}\left(M_{N}\right)$, would imply that $v^{\{x, y\}}\left(M_{N}\right)=$ $\{x, y\}$. Hence, by consistency in alternatives, $x \in v^{\{x, y, z\}}\left(M_{N}\right)$, which contradicts case (a). 
(c) $z \notin v^{\{x, y, z\}}\left(M_{N}\right)$. If it was the case that $z \in v^{\{x, y, z\}}\left(M_{N}\right)$, then, by consistency in alternatives, we would have $z \in v^{\{y, z\}}\left(M_{N}\right)$. This, together with the assumption $y \in v^{\{y, z\}}\left(M_{N}\right)$, would imply that $v^{\{y, z\}}\left(M_{N}\right)=$ $\{y, z\}$. Hence, by consistency in alternatives, $y \in v^{\{x, y, z\}}\left(M_{N}\right)$, which contradicts case (b).

The three cases together imply that $v^{\{x, y, z\}}\left(M_{N}\right)=\emptyset$. This is not possible by definition and, therefore, we have reached a contradiction. Consequently, $\succsim$ is transitive. Since the binary relation $\succsim$ is well-defined, complete, and transitive, it is a complete preorder.

Associativity: The property holds because + , the usual addition operator on vectors, is associative.

INDEPENDENCE: Consider any triple $\left(x_{1}, \ldots, x_{\theta}\right),\left(y_{1}, \ldots, y_{\theta}\right),\left(z_{1}, \ldots, z_{\theta}\right) \in$ $\mathbb{N}^{\theta}$ such that $\left(x_{1}, \ldots, x_{\theta}\right) \succsim\left(y_{1}, \ldots, y_{\theta}\right)$. Take any profile $M$, any two disjoint electorates $A, B \subset I$, and any two alternatives $x, y \in X$ such that for all $t \in \Theta, G_{x}^{t}\left(M_{A}\right)=x_{t}, G_{y}^{t}\left(M_{A}\right)=y_{t}$, and $G_{x}^{t}\left(M_{B}\right)=G_{y}^{t}\left(M_{B}\right)=z_{t}$. Since $\left(x_{1}, \ldots, x_{\theta}\right) \succsim\left(y_{1}, \ldots, y_{\theta}\right)$ by assumption, the definition of $\succsim$ implies that $x \in v^{\{x, y\}}\left(M_{A}\right)$. Also, $v^{\{x, y\}}\left(M_{B}\right)=\{x, y\}$ by Lemma 2. By consistency in voters, $x \in v^{\{x, y\}}\left(M_{A \cup B}\right)$. Given that $G_{x}^{t}\left(M_{A \cup B}\right)=x_{t}+z_{t}$ and $G_{y}^{t}\left(M_{A \cup B}\right)=y_{t}+z_{t}$ for all $t \in \Theta$, it follows from the definition of $\succsim$ that $\left(x_{1}+z_{1}, \ldots, x_{\theta}+z_{\theta}\right) \succsim\left(y_{1}+z_{1}, \ldots, y_{\theta}+z_{\theta}\right)$. Hence, as desired, $\left(\left(x_{1}, \ldots, x_{\theta}\right)+\left(z_{1}, \ldots, z_{\theta}\right)\right) \succsim\left(\left(y_{1}, \ldots, y_{\theta}\right)+\left(z_{1}, \ldots, z_{\theta}\right)\right)$ and $\left(\left(z_{1}, \ldots, z_{\theta}\right)+\right.$ $\left.\left(x_{1}, \ldots, x_{\theta}\right)\right) \succsim\left(\left(z_{1}, \ldots, z_{\theta}\right)+\left(y_{1}, \ldots, y_{\theta}\right)\right)$.

Archimedean: Take any $\left(x_{1}, \ldots, x_{\theta}\right),\left(y_{1}, \ldots, y_{\theta}\right),\left(z_{1}, \ldots, z_{\theta}\right),\left(w_{1}, \ldots, w_{\theta}\right)$ belonging to $\mathbb{N}^{\theta}$ such that $\left(x_{1}, \ldots, x_{\theta}\right) \succ\left(y_{1}, \ldots, y_{\theta}\right)$. Consider any profile $M$, any two alternatives $x, y \in X$, any electorate $A \subset I$, and any succes- 
sion of disjoint electorates $\left\{N_{p}\right\}_{p \in \mathbb{N}}$ such that $G_{x}^{t}\left(M_{A}\right)=z_{t}, G_{y}^{t}\left(M_{A}\right)=w_{t}$, $v^{\{x, y\}}\left(N_{p}\right)=\{x\}, A \cap N_{p}=\emptyset, G_{x}^{t}\left(M_{N_{p}}\right)=x_{t}$ and $G_{y}^{t}\left(M_{N_{p}}\right)=y_{t}$ for all $p \in \mathbb{N}$. By continuity, there exists $k \in \mathbb{N}$ such that $x \in v^{\{x, y\}}\left(M_{N_{1} \cup \ldots \cup N_{k} \cup A}\right)$. Since $G_{x}^{t}\left(M_{N_{1} \cup \ldots \cup N_{k} \cup A}\right)=k \cdot x_{t}+z_{t}$ and $G_{y}^{t}\left(M_{N_{1} \cup \ldots \cup N_{k} \cup A}\right)=k \cdot y_{t}+w_{t}$ for all $t \in \Theta$, we have, as desired, that $\left(k \cdot\left(x_{1}, \ldots, x_{\theta}\right)+\left(z_{1}, \ldots, z_{\theta}\right)\right) \succsim$ $\left(k \cdot\left(y_{1}, \ldots, y_{\theta}\right)+\left(w_{1}, \ldots, w_{\theta}\right)\right)$.

Since the triple $\left(\mathbb{N}^{\theta}, \succsim,+\right)$ is a closed extensive structure, we can apply Theorem 1 in Krantz et al. (1971) which guarantees that there exists a real-valued function $f$ over $\mathbb{N}^{\theta}$ such that for all $\left(x_{1}, \ldots, x_{\theta}\right),\left(y_{1}, \ldots, y_{\theta}\right) \in \mathbb{N}^{\theta}$ :

(i) $\left(x_{1}, \ldots, x_{\theta}\right) \succsim\left(y_{1}, \ldots, y_{\theta}\right) \Leftrightarrow f\left(x_{1}, \ldots, x_{\theta}\right) \geq f\left(y_{1}, \ldots, y_{\theta}\right)$ and

(ii) $f\left(\left(x_{1}, \ldots, x_{\theta}\right)+\left(y_{1}, \ldots, y_{\theta}\right)\right)=f\left(x_{1}, \ldots, x_{\theta}\right)+f\left(y_{1}, \ldots, y_{\theta}\right)$.

Additionally, any other function $g$ satisfies conditions $(i)$ and $(i i)$ if and only if there exists $t \in \mathbb{R}_{++}$such that $g=t \cdot f$.

Using this result we construct the vector of weights $\mathbf{p}=\left(p_{1}, \ldots, p_{\theta}\right)$ by setting $f(1,0, \ldots, 0)$ equal to $p_{1}, f(0,1,0, \ldots, 0)$ equal to $p_{2}$, and so forth. Since we know from condition (ii) that $f\left(x_{1}, \ldots, x_{\theta}\right)=f\left(x_{1}, 0, \ldots, 0\right)+$ $f\left(0, x_{2}, 0, \ldots, 0\right)+\ldots+f\left(0,0, \ldots, x_{\theta}\right)$, we have that

$$
f\left(x_{1}, \ldots, x_{\theta}\right) \geq f\left(y_{1}, \ldots, y_{\theta}\right) \Leftrightarrow \sum_{t=1}^{\theta} p_{t} \cdot x_{t} \geq \sum_{t=1}^{\theta} p_{t} \cdot y_{t} .
$$

Then, it follows from condition (i) and the definition of $\succsim$ that for all response profiles $M_{N}$ and all alternatives $x, y \in X$,

$$
x \in v^{\{x, y\}}\left(M_{N}\right) \Leftrightarrow \sum_{t=1}^{\theta} p_{t} \cdot G_{x}^{t}\left(M_{N}\right) \geq \sum_{t=1}^{\theta} p_{t} \cdot G_{y}^{t}\left(M_{N}\right) .
$$

We also know from Faithfulness that $p_{t}>0$ for all $t \in \Theta$ and, therefore, we have shown that the subfamily $\left\{v^{K}\right\}_{|K|=2}$ is a Type-weighted Approval Voting with respect to the vector of weights $\mathbf{p}=\left(p_{1}, p_{2}, \ldots, p_{\theta}\right)$. Consequently, 
it remains to be shown that the vector of weights $\mathbf{p}=\left(p_{1}, p_{2}, \ldots, p_{\theta}\right)$ is such that for all sets of feasible alternatives $K \subseteq X$, independently of its size, and all response profiles $M_{N}$,

$x \in v^{K}\left(M_{N}\right)$ if and only if $\sum_{t \in \Theta} p_{t} \cdot G_{x}^{t}\left(M_{N}\right) \geq \sum_{t \in \Theta} p_{t} \cdot G_{y}^{t}\left(M_{N}\right)$ for all $y \in K$.

Suppose first that $x \in v^{K}\left(M_{N}\right)$. Then, by consistency in alternatives, $x \in v^{\{x, y\}}\left(M_{N}\right)$ for all $y \in K \backslash\{x\}$. Since we already know that, for all pairs of alternatives, $v^{\{x, y\}}$ is the Type-weighted Approval Voting with respect to $\mathbf{p}=\left(p_{1}, p_{2}, \ldots, p_{\theta}\right)$, it has to be the case that $\sum_{t \in \Theta} p_{t} \cdot G_{x}^{t}\left(M_{N}\right) \geq \sum_{t \in \Theta} p_{t}$. $G_{y}^{t}\left(M_{N}\right)$ for all $y \in K$.

Suppose now that $\sum_{t \in \Theta} p_{t} \cdot G_{x}^{t}\left(M_{N}\right) \geq \sum_{t \in \Theta} p_{t} \cdot G_{y}^{t}\left(M_{N}\right)$ for all $y \in$ $K$. Then, given that, for all pairs of alternatives, $v^{\{x, y\}}\left(M_{N}\right)$ is the Typeweighted Approval Voting with respect to $\mathbf{p}=\left(p_{1}, p_{2}, \ldots, p_{\theta}\right), x \in v^{\{x, y\}}\left(M_{N}\right)$ for all $y \in K \backslash\{x\}$. If there is some $z \neq x$ such that $z \in v^{K}\left(M_{N}\right)$, then $v^{K}\left(M_{N}\right) \cap\{x, z\} \neq \emptyset$ and it follows from consistency in alternatives that $v^{\{x, z\}}\left(M_{N}\right)=v^{K}\left(M_{N}\right) \cap\{x, z\}$. Since we have already seen that $x \in$ $v^{\{x, z\}}\left(M_{N}\right)$ it also has to be that $x \in v^{K}\left(M_{N}\right)$. Finally, if there is no $z \neq x$ such that $z \in v^{K}\left(M_{N}\right)$, then $v^{K}\left(M_{N}\right)=\{x\}$ because $v^{K}\left(M_{N}\right) \neq \emptyset$. This concludes the proof of the theorem.

\section{Proof of Proposition 1}

To establish the independence of the axioms, we are going to present six voting procedures that violate one different axiom each and satisfy the remaining five properties.

Consistency in alternatives: Take any type $t \in \Theta$. Let the voting procedure $v$ be equal to Approval Voting whenever the set of feasible alternatives 
$K$ contains exactly two alternatives; otherwise, apply the Type-weighted Approval Voting with weights $p_{t}=1$ and $p_{s}=2$ for all types $s \neq t$. This procedure is consistent in voters, type--wise anonymous, neutral, faithful, and continuous. The following example shows that it is not consistent in alternatives.

Consider $X=\{x, y, z\}$ and suppose that $i \in I_{s}$ and $j \in I_{t}$. If $M_{i}=\{x\}$ and $M_{j}=\{y\}$, then $v^{X}\left(M_{\{i, j\}}\right)=\{x\}$ and $v^{\{x, y\}}\left(M_{\{i, j\}}\right)=\{x, y\}$. Since $v^{X}\left(M_{\{i, j\}}\right) \cap\{x, y\} \neq \emptyset$, consistency in alternatives implies that $v^{\{x, y\}}\left(M_{\{i, j\}}\right)=$ $v^{X}\left(M_{\{i, j\}}\right) \cap\{x, y\}=\{x\}$. This contradicts that $v^{\{x, y\}}\left(M_{\{i, j\}}\right)=\{x, y\}$.

Consistency in voters: Let the voting procedure $v$ be equal to Approval Voting whenever all individuals belonging to the electorate $N$ are of the same type; otherwise select all feasible alternatives. This procedure is consistent in alternatives, type-wise anonymous, neutral, faithful, and continuous. The following example shows that it is not consistent in voters.

Consider $X=\{x, y\}$ and suppose that $1 \in I_{s}$ and $2 \in I_{t}$. If $M_{1}=M_{2}=$ $\{x\}$, then $v^{\{x, y\}}\left(M_{1}\right)=v^{\{x, y\}}\left(M_{2}\right)=\{x\}$ and $v^{\{x, y\}}\left(M_{\{1,2\}}\right)=\{x, y\}$. Since $v^{\{x, y\}}\left(M_{1}\right) \cap v^{\{x, y\}}\left(M_{2}\right) \neq \emptyset$, consistency in voters implies that $v^{\{x, y\}}\left(M_{\{1,2\}}\right)=$ $\{x\}$. This contradicts that $v^{\{x, y\}}\left(M_{\{1,2\}}\right)=\{x, y\}$.

Type-wise anonymity: Assign to each individual $i \in I$ a weight $p_{i}$ greater than a strictly positive number $\epsilon$. Also assume that $p_{i}>p_{j}$ for some pair $i, j \in I_{t}$ for some $t \in \Theta$. Let the voting procedure $v$ be such that for all sets of feasible alternatives $K \subseteq X$, all profiles $M \in\left(2^{X}\right)^{I}$, and all electorates $N \subset I, x \in v^{K}\left(M_{N}\right)$ if and only if $\sum_{i \in N: x \in M_{i}} p_{i} \geq \sum_{i \in N: y \in M_{i}} p_{i}$ for all $y \in K$. This procedure is consistent in alternatives, consistent in voters, neutral, faithful, and continuous. The following example shows that it is not type-wise anonymous. 
Consider $X=\{x, y\}$ and $i, j \in I_{t}$ for some $t \in \Theta$ such that $p_{i}>p_{j}$. If $M_{i}=\{x\}$ and $M_{j}=\{y\}$, then $v^{\{x, y\}}\left(M_{\{i, j\}}\right)=\{x\}$. Now, take any permutation $\pi_{t}: I_{t} \rightarrow I_{t}$ such that $\pi_{t}(i)=j$ and $\pi_{t}(j)=i$. Then, $v^{\{x, y\}}\left(M_{\left\{\pi_{t}(i), \pi_{t}(j)\right\}}\right)=\{y\}$. Since type-wise anonymity implies that $v^{\{x, y\}}$ $\left(M_{\left.\pi_{t}(i), \pi_{t}(j)\right\}}\right)=v^{\{x, y\}}\left(M_{\{i, j\}}\right)$, this is a contradiction.

Neutrality: Assign to each alternative $x \in X$ a strictly positive weight $p_{x}$. Assume also that $p_{x}>p_{y}$ for some $x, y \in X$. Let the voting procedure $v$ be such that for all sets of feasible alternatives $K \subseteq X$, all profiles $M \in$ $\left(2^{X}\right)^{I}$, and all electorates $N \subset I, x \in v^{K}\left(M_{N}\right)$ if and only if $p_{x} \cdot G_{x}\left(M_{N}\right) \geq$ $p_{y} \cdot G_{y}\left(M_{N}\right)$ for all $y \in K$. This procedure is consistent in alternatives, consistent in voters, type-wise anonymous, faithful and continuous. The following example shows that it is not neutral.

Consider $X=\{x, y\}$ and $N=\{i, j\}$ and suppose that $p_{x}<p_{y}$. If $M_{i}=\{x\}$ and $M_{j}=\{y\}$, then $v^{\{x, y\}}\left(M_{N}\right)=\{y\}$. Now let the permutation $\mu: X \rightarrow X$ be such that $\mu(x)=y$ and $\mu(y)=x$. Then, $\mu\left(v^{\{x, y\}}\left(M_{N}\right)\right)=\{x\}$ and $v^{\mu(\{x, y\})}\left(\mu\left(M_{N}\right)\right)=\{y\}$. Since neutrality implies that $\mu\left(v^{\{x, y\}}\left(M_{N}\right)\right)=v^{\mu(\{x, y\})}\left(\mu\left(M_{N}\right)\right)$, this is a contradiction.

Faithfulness: Let the voting procedure $v$ be such that for all sets of feasible alternatives $K \subseteq X$, all profiles $M \in\left(2^{X}\right)^{I}$, and all electorates $N \subset I$, $v^{K}\left(M_{N}\right)=K$. This procedure is consistent in alternatives, consistent in voters, type--wise anonymous, neutral, and continuous. The following example shows that it is not faithful.

Consider $X=\{x, y\}$ and $N=\{i\}$. Suppose that $M_{i}=\{x\}$. Then, $v^{\{x, y\}}\left(M_{i}\right)=\{x, y\}$. However, faithfulness implies that $v^{\{x, y\}}\left(M_{i}\right)=M_{i} \cap$ $\{x, y\}=\{x\}$, which is a contradiction.

Continuity: Take any vector $\mathbf{q}=\left(q_{1}, q_{2}, \ldots, q_{\theta}\right)$ of strictly positive weights 
such that $q_{i} \neq q_{j}$ for some $i, j \in \Theta$. Let the voting procedure $v$ be such that for all sets of feasible alternatives $K \subseteq X$, all profiles $M \in\left(2^{X}\right)^{I}$, and all electorates $N \subset I, x \in v^{K}\left(M_{N}\right)$ if and only if (a) $G_{x}\left(M_{N}\right) \geq G_{y}\left(M_{N}\right)$ for all $y \in K$ and (b) $\sum_{t \in \Theta} q_{t} \cdot G_{x}^{t}\left(M_{N}\right) \geq \sum_{t \in \Theta} q_{t} \cdot G_{y}^{t}\left(M_{N}\right)$ for all $y \in K$ such that $G_{x}\left(M_{N}\right)=G_{y}\left(M_{N}\right)$. This procedure is consistent in alternatives, consistent in voters, type-wise anonymous, neutral, and faithful. The following example shows that it is not continuous.

Consider $X=\{x, y\}, I_{s}=\left\{j_{i}\right\}_{i \in \mathbb{N}}, I_{t}=\left\{k_{i}\right\}_{i \in \mathbb{N}}$ such that $q_{s}>q_{t}$. Suppose that $M_{j}=\{x\}$ for all $j \in I_{s}$ and $M_{k}=\{y\}$ for all $k \in I_{t}$. Suppose additionally that $M_{l}=\{y\}$ for some $l \in I_{t}$. Consider the electorates $N_{i}=\left\{j_{i}, k_{i}\right\}$ for all $i \in \mathbb{N}$. By the definition of $v, v^{\{x, y\}}\left(M_{l}\right)=\{y\}$ and $v^{\{x, y\}}\left(M_{N_{i}}\right)=\{x\}$ for all $i \in \mathbb{N}$. Consequently, continuity implies that there is some $b \in \mathbb{N}$ such that $x \in v^{\{x, y\}}\left(M_{N_{1} \cup N_{2} \cup \ldots \cup N_{b} \cup\{l\}}\right)$. However, since $G_{y}\left(M_{N_{1} \cup N_{2} \cup \ldots \cup N_{b} \cup\{l\}}\right)>$ $G_{x}\left(M_{N_{1} \cup N_{2} \cup \ldots \cup N_{b} \cup\{l\}}\right)$ for all $b \in \mathbb{N}, v^{\{x, y\}}\left(M_{N_{1} \cup N_{2} \cup \ldots \cup N_{b} \cup\{l\}}\right)=\{y\}$ for all $b \in \mathbb{N}$. This is a contradiction.

\section{Proof of Theorem 2}

It is easy to check that Approval and Disapproval Voting satisfy consistency and symmetry. The proof that these properties imply $v$ to be one of these voting procedures follows the steps mentioned in the main text.

Observe that Lemma 1 still applies. The following lemma introduces a stronger result than that of Lemma 3, given the strengthening of weak symmetry to symmetry.

Lemma 5 If the voting procedure $v$ is consistent in voters and symmetric, then for all alternatives $x, y, z, w \in X$, all profiles $M, M^{\prime} \in\left(2^{X}\right)^{I}$, and all 
electorates $A, B \subset I$ such that $G_{x}\left(M_{A}\right)=G_{z}\left(M_{B}^{\prime}\right)$ and $G_{y}\left(M_{A}\right)=G_{w}\left(M_{B}^{\prime}\right)$, $x \in v^{\{x, y\}}\left(M_{A}\right) \Leftrightarrow z \in v^{\{z, w\}}\left(M_{B}^{\prime}\right)$ and $y \in v^{\{x, y\}}\left(M_{A}\right) \Leftrightarrow w \in v^{\{z, w\}}\left(M_{B}^{\prime}\right)$.

Proof: Take any four alternatives $x, y, z, w \in X$, any two profiles $M, M^{\prime} \in$ $\left(2^{X}\right)^{I}$, and any two electorates $A, B \subset I$ such that $G_{x}\left(M_{A}\right)=G_{z}\left(M_{B}^{\prime}\right)$ and $G_{y}\left(M_{A}\right)=G_{w}\left(M_{B}^{\prime}\right)$. By Lemma 1 , we can assume that $M_{i} \cap\{x, y\} \in$ $\{\{x\},\{y\}\}$ for all $i \in A$ and $M_{j}^{\prime} \cap\{x, y\} \in\{\{x\},\{y\}\}$ for all $j \in B$. Consider the permutation $\mu: X \rightarrow X$ such that $\mu(z)=x$ and $\mu(w)=y$. Then, it is easy to see that $\mu\left(M_{B}^{\prime}\right)$ and $M_{A}$ are strongly isomorphic relative to $\{x, y\}$. Then, by anonymity,

$$
v^{\{x, y\}}\left(M_{A}\right)=v^{\{x, y\}}\left(\mu\left(M_{B}^{\prime}\right)\right) .
$$

Finally, consider the permutation $\mu^{-1}$. By neutrality, $\mu^{-1}\left(v^{\{x, y\}}\left(\mu\left(M_{B}^{\prime}\right)\right)\right)=$ $v^{\mu^{-1}(\{x, y\})}\left(\mu^{-1}\left(\mu\left(M_{B}^{\prime}\right)\right)\right)=v^{\{z, w\}}\left(M_{B}^{\prime}\right)$. This, together with Equation (4), implies that $\mu^{-1}\left(v^{\{x, y\}}\left(M_{A}\right)\right)=v^{\{z, w\}}\left(M_{B}^{\prime}\right)$. Hence, $x \in v^{\{x, y\}}\left(M_{A}\right)$ if and only if $z \in v^{\{z, w\}}\left(M_{B}^{\prime}\right)$ and $y \in v^{\{x, y\}}\left(M_{A}\right)$ if and only if $w \in v^{\{z, w\}}\left(M_{B}^{\prime}\right)$.

Now, we introduce a binary relation $\succsim$ defined over $\mathbb{N} \times \mathbb{N}$ : for all $a, b \in \mathbb{N}$, $a \succsim b$ if there exists a response profile $M_{N}$ and two alternatives $x, y \in X$ such that $x \in v^{\{x, y\}}\left(M_{N}\right), G_{x}\left(M_{N}\right)=a$ and $G_{y}\left(M_{N}\right)=b$.

Lemma 6 The binary relation $\succsim i s \geq, \leq$ or $=$.

Proof: First, it is easy to see that, by Lemma $5, \succsim$ is well-defined. The proof that it is complete and transitive follows the same steps as the corresponding proof of Theorem 1. Thus, it is omitted.

Since the binary relation $\succsim$ is well-defined, complete, and transitive, it is a complete preorder. Now, we will show that for all $a, b \in \mathbb{N}, a \succsim b$ if and only if $(a+1) \succsim(b+1)$. To prove this, consider a response profile $M_{N}$ such 
that $G_{x}\left(M_{N}\right)=a$ and $G_{y}\left(M_{N}\right)=b$ and two individuals $i, j \in(I \backslash N)$ such that $M_{i} \cap\{x, y\}=\{x\}$ and $M_{j} \cap\{x, y\}=\{y\}$.

Assume first that $a \succsim b$. Then, by definition of $\succsim$, we have that $x \in$ $v^{\{x, y\}}\left(M_{N}\right)$ and, by reflexivity of $\succsim, v^{\{x, y\}}\left(M_{\{i, j\}}\right)=\{x, y\}$. Finally, apply consistency in voters to see that $x \in v^{\{x, y\}}\left(M_{N \cup\{i, j\}}\right)$. Thus, $(a+1) \succsim(b+1)$.

Similarly, assume now that $(a+1) \succsim(b+1)$. Then, by definition of $\succsim$, we have that $x \in v^{\{x, y\}}\left(M_{N \cup\{i, j\}}\right)$ and, by reflexivity of $\succsim, v^{\{x, y\}}\left(M_{\{i, j\}}\right)=$ $\{x, y\}$. Finally, use consistency in voters and the fact that $v^{\{x, y\}}\left(M_{N}\right) \neq \emptyset$ by definition, to see that $x \in v^{\{x, y\}}\left(M_{N}\right)$. Thus, $a \succsim b$.

Therefore, it is easy to see that $\succsim$ must be $\geq, \leq$ or $=$.

It follows from Lemma 6 and the definition of $\succsim$ that if the subfamily $\left\{v^{K}\right\}_{|K|=2}$ is non-degenerate, it must be Approval or Disapproval Voting. The proof that this also occurs independently of the size of $K$ follows the same steps than in the proof of Theorem 1 and is thus omitted. This concludes the proof of the theorem. 


\begin{tabular}{|lcc|}
\hline EU Member State & Population & Weight \\
\hline \hline Germany & $81,757,595$ & 29 \\
France & $64,709,480$ & 29 \\
United Kingdom & $62,041,708$ & 29 \\
Italy & $60,397,353$ & 29 \\
Spain & $46,087,170$ & 27 \\
Poland & $38,163,895$ & 27 \\
Romania & $21,466,174$ & 14 \\
Netherlands & $16,576,800$ & 13 \\
Greece & $11,125,179$ & 12 \\
Belgium & $10,827,519$ & 12 \\
Portugal & $10,636,888$ & 12 \\
Czech Republic & $10,512,397$ & 12 \\
Hungary & $10,013,628$ & 12 \\
Sweden & $9,372,899$ & 10 \\
Austria & $8,372,930$ & 10 \\
Bulgaria & $7,576,751$ & 10 \\
Denmark & $5,547,088$ & 7 \\
Slovakia & $5,424,057$ & 7 \\
Finland & $5,350,475$ & 7 \\
Ireland & $4,450,878$ & 7 \\
Lithuania & $3,329,227$ & 7 \\
Latvia & $2,248,961$ & 4 \\
Slovenia & $2,054,119$ & 4 \\
Estonia & $1,340,274$ & 4 \\
Cyprus & 801,851 & 4 \\
Luxembourg & 502,207 & 4 \\
Malta & 416,333 & 3 \\
\hline
\end{tabular}

Table 1: Voting Weights in the EU Member State Council as of July 2011. 


\begin{tabular}{|lcc|}
\hline IMF Board of Directors & Percentage of Fund & Weight \\
\hline \hline United States & $16.77 \%$ & 421,964 \\
Japan & $6.24 \%$ & 157,025 \\
Germany & $5.82 \%$ & 146,395 \\
France & $4.30 \%$ & 108,125 \\
United Kingdom & $4.30 \%$ & 108,125 \\
Belgium (Austria) & $4.98 \%$ & 125,221 \\
Mexico (Venezuela) & $4.65 \%$ & 117,053 \\
Netherlands (Ukraine) & $4.52 \%$ & 113,822 \\
Italy (Greece) & $4.26 \%$ & 107,077 \\
Singapore (Indonesia) & $3.94 \%$ & 99,062 \\
China & $3.82 \%$ & 95,999 \\
Australia (Korea) & $3.63 \%$ & 91,347 \\
Canada (Ireland) & $3.61 \%$ & 90,708 \\
Denmark (Norway) & $3.39 \%$ & 85,352 \\
Lesotho (Gambia) & $3.22 \%$ & 81,085 \\
Egypt (Lebanon) & $3.13 \%$ & 78,692 \\
India (Sri Lanka) & $2.81 \%$ & 70,705 \\
Saudi Arabia & $2.81 \%$ & 70,595 \\
Brazil (Colombia) & $2.79 \%$ & 70,188 \\
Switzerland (Poland) & $2.78 \%$ & 69,842 \\
Russian Federation & $2.36 \%$ & 60,194 \\
Iran (Morocco) & $2.27 \%$ & 57,092 \\
Argentina (Chile) & $1.84 \%$ & 46,335 \\
Togo (Chad) & $1.55 \%$ & 39,039 \\
\hline
\end{tabular}

Table 2: Voting Weights in the Board of Directors of the IMF as of July 2011. One voter tends to represent several countries, the only exceptions are the United States, Japan, Germany, France, United Kingdom, China, and the Russian Federation. For example, Argentina is grouped with Bolivia, Chile, Paraguay, Peru, and Uruguay. In case the Argentinian representant is absent, the Chilean representant replaces her/him. The exact categorization can be consulted at http://www.imf.org/external/np/sec/memdir/eds.aspx. 